\title{
USO DO ATRIBUTO AMPLITUDE INSTANTÂNEA DO DADO GPR NA AVALIAÇÃO DO TEOR DE UMIDADE DE SOLOS E SEDIMENTOS
}

\author{
Renato Luiz Prado ${ }^{1}$, Emílio Eduardo Moreira Barbosa², Rodolfo Moreda Mendes ${ }^{3}$, \\ Fernando Antônio Medeiros Marinho ${ }^{4}$ e Orlando Martini de Oliveira ${ }^{5}$
}

Recebido em 10 março, 2011 / Aceito em 22 agosto, 2011

Received on March 10, 2011 / Accepted on August 22, 2011

\begin{abstract}
This paper presents results of a study conducted on laboratory and field in order to analyze the parameter amplitude of electromagnetic wave in qualitative assessment of soil moisture contents. We obtained empirical correlations between instantaneous amplitude of the wave, recorded by GPR - Ground Penetrating Radar, and the values of volumetric sediment moisture contents, obtained by TDR - Time Domain Reflectometer, in laboratory experiment as well as in soil samples in a field experiment. Results showed that there is a strong correlation between environmental moisture contents and instantaneous amplitude parameter of electromagnetic wave. In controlled laboratory conditions, this correlation showed a strong coefficient of determination. However, in the field experiment, the coefficient was only 0.64 due to the small amount of data used. This method is easy to implement through a processing flowchart of rapid execution, and can be used in addition to the velocity attribute usually employed in these correlations.
\end{abstract}

Keywords: GPR, moisture content, instantaneous amplitude, TDR, soils.

RESUMO. Este trabalho apresenta os resultados de um estudo realizado em escala de laboratório e de campo visando à análise do parâmetro amplitude da onda eletromagnética na avaliação qualitativa dos teores de umidade dos solos. Foram obtidas correlações empíricas entre o parâmetro amplitude instantânea da onda, registrada pelo radar de penetração no solo - GPR, e os valores de umidade volumétrica de sedimentos obtidos por reflectômetro no domínio do tempo - TDR, em experimento de laboratório, como também de amostras de solos, em experimento de campo. Os resultados indicaram que há forte correspondência entre os teores de umidade do meio e o parâmetro amplitude instantânea da onda eletromagnética. Em condições controladas de laboratório, a correlação apresentou forte coeficiente de determinação, mas no experimento de campo o coeficiente foi de apenas 0,64 devido ao pequeno número de dados empregados na correlação. 0 método é de fácil implementação, através de um fluxograma de processamento de rápida execução, e seus resultados podem ser usados complementarmente aos obtidos da análise do atributo velocidade.

Palavras-chave: GPR, teor de umidade, amplitude instantânea, TDR, solos.

\footnotetext{
${ }^{1}$ Instituto de Astronomia, Geofísica e Ciências Atmosféricas, Universidade de São Paulo, Rua do Matão, 1226, Cidade Universitária, 05508-090 São Paulo, SP, Brasil. Tel.: (11) 3091-2762; Fax: (11) 3091-5034 - E-mail: renato@iag.usp.br

2 Programa de Pós-graduação, Instituto de Astronomia, Geofísica e Ciências Atmostéricas, Universidade de São Paulo, Rua do Matão, 1226, Cidade Universitária, 05508-090 São Paulo, SP, Brasil. Tel.: (11) 3091-2789; Fax: (11) 3091-5094 - E-mail: emilio@iag.usp.br

3 Instituto Geológico do Estado de São Paulo, Avenida Miguel Stéfano, 3900, Água Funda, Caixa Postal 8772, 04301-903 São Paulo, SP, Brasil. Tel.: (11) 5058-9994; Ramal: 2047; Fax: (11) 5073-5511 - E-mail: rodolfommendes@ig.com.br

${ }^{4}$ Escola Politécnica, Universidade de São Paulo, Avenida Professor Almeida Prado, 271, Cidade Universitária, 05508-900 São Paulo, SP, Brasil. Tel.: (11) 3091-5703: Fax: (11) 3818-5181-E-mail: fmarinho@usp.br

${ }^{5}$ Departamento de Engenharia Civil/CTC, Universidade Federal de Santa Catarina, Rua João Pio Duarte da Silva, 205, 88040-970 Florianópolis, SC, Brasil. Tel.: (48) 3721-7761; Fax: (48) 3331-5191 - E-mail: martini@ecv.ufsc.br
} 


\section{INTRODUÇÃo}

0 mapeamento da variação do conteúdo de água da subsuperfície rasa é importante em diversas áreas de aplicação como na agronomia, para o controle dos processos de irrigação (Grote et al., 2003), ou na geotecnia, para subsidiar estudos de instabilidade de encostas (Kim et al., 2004).

0 GPR (Ground Penetrating Radar - radar de penetração no solo) tem sido empregado nessas predições por ser uma técnica não destrutiva e de alta resolução vertical, como também pela rapidez do levantamento. Outro método empregado e muitas vezes coadjuvante importante nesses estudos, o TDR ( Time Domain Reflectometry), tem como desvantagem, relativamente ao GPR, 0 fato de representar uma resposta pontual, ser invasivo, e requerer, idealmente, calibrações específicas para os diferentes tipos de solos.

Nessas aplicações, o método GPR, na maioria das vezes, se utiliza do registro da onda refletida na interface com a zona saturada ou entre estratos com diferentes propriedades elétricas, normalmente empregando arranjos CMP - Common Mid Point (Huisman et al., 2003), que consomem muito tempo na aquisição e não permitem a obtenção de seções contínuas.

Há diversos fatores que influenciam a acurácia da medida com GPR em relação à estimativa do teor de umidade do meio, mas o principal deles está relacionado à etapa da determinação da velocidade de propagação da onda. Uma aproximação da constante dielétrica é obtida da velocidade, e a partir daquela derivase 0 teor de umidade volumétrico, usualmente empregando a equação empírica de Topp et al. (1980).

Ao contrário do que ocorre em relação ao parâmetro velocidade, não há muitos trabalhos apresentando resultados de correlações entre o parâmetro amplitude do sinal de radar e teores de umidade (Charlton, 2000; Corbeanu et al., 2002; Schmalz et al., 2002). Alterações nas propriedades petrofísicas do meio relacionadas às variações dos teores de umidade nos poros se traduzem em diferenças nas propriedades elétricas do meio (permissividade dielétrica e condutividade elétrica), e consequentemente em mudanças nos atributos da onda no seu percurso. Pettinelli et al. (2007), restringindo a análise do atributo amplitude à janela temporal que contém apenas o registro da onda direta, sugeriram seu emprego para a estimativa da variação espacial do teor de umidade da subsuperfície rasa.

Neste trabalho apresentamos os resultados de um estudo realizado em escala de laboratório e de campo visando à análise do parâmetro amplitude para avaliar qualitativamente os teores de umidade dos solos. São apresentados resultados de correlações empíricas obtidas entre 0 parâmetro amplitude instantânea da onda direta registrada pelo GPR e 0 teor de umidade volumétrica de sedimentos obtidos por TDR, em experimento de laboratório, assim como entre o parâmetro amplitude e 0 teor de umidade obtido de amostra de solo, em experimento de campo. Analisamos a onda relacionada à propagação direta entre as antenas transmissora e receptora pela facilidade de registro e identificação em levantamentos convencionais com afastamento (offset) constante. Ademais, o evento onda direta é confinado ao limite da superfície ar-solo e a profundidades de influência normalmente inferiores a um metro (dependendo da frequência central das antenas), limites importantes nas avaliações dos processos de infiltração e escoamento superficial.

Apresentamos apenas observações quanto à influência do parâmetro teor de umidade, uma vez que os experimentos foram realizados considerando sempre o mesmo meio.

No experimento de laboratório utilizou-se um tanque com areia com sistema de injeção e drenagem de água, e no experimento de campo foram realizados ensaios ao longo de um período de 12 meses sempre no mesmo local. Como a amplitude da resposta GPR também está relacionada a outras propriedades petrofísicas do meio, como composição mineralógica, tamanho dos grãos e características dos poros, principalmente, há a necessidade de se desenvolver estudos de modelagem que incluam essas outras propriedades para que possam ser estabelecidas relações quantitativas entre amplitude instantânea $\mathrm{e}$ teor de umidade.

\section{METODOLOGIA}

\section{Experimento de laboratório}

Visando à simulação da zona vadosa em duas condições distintas de umidade, preencheu-se um tanque cilíndrico de fibra de vidro (2,1 m de altura e 1,4 m de diâmetro) com areia selecionada. Um dispositivo instalado na sua base permitiu a injeção de água até a completa saturação do meio e o posterior esgotamento do tanque (Fig. 1). Pares de sensores TDR diametralmente opostos foram dispostos ao longo da parede do tanque.

Com uma antena blindada de $1000 \mathrm{MHz}$ de frequência central (afastamento fixo entre as antenas de 0,105 $\mathrm{m}$ ) foram adquiridos perfis no modo transversal elétrico - TE ao longo da linha diametral do tanque (L1) antes do enchimento, após a completa saturação, e logo depois de seu esvaziamento. Da mesma forma foram adquiridos dados com a antena fixa (posicionada no final da seção L1) com registros a cada 2 segundos durante todo 0 tempo do enchimento e esvaziamento do tanque. 

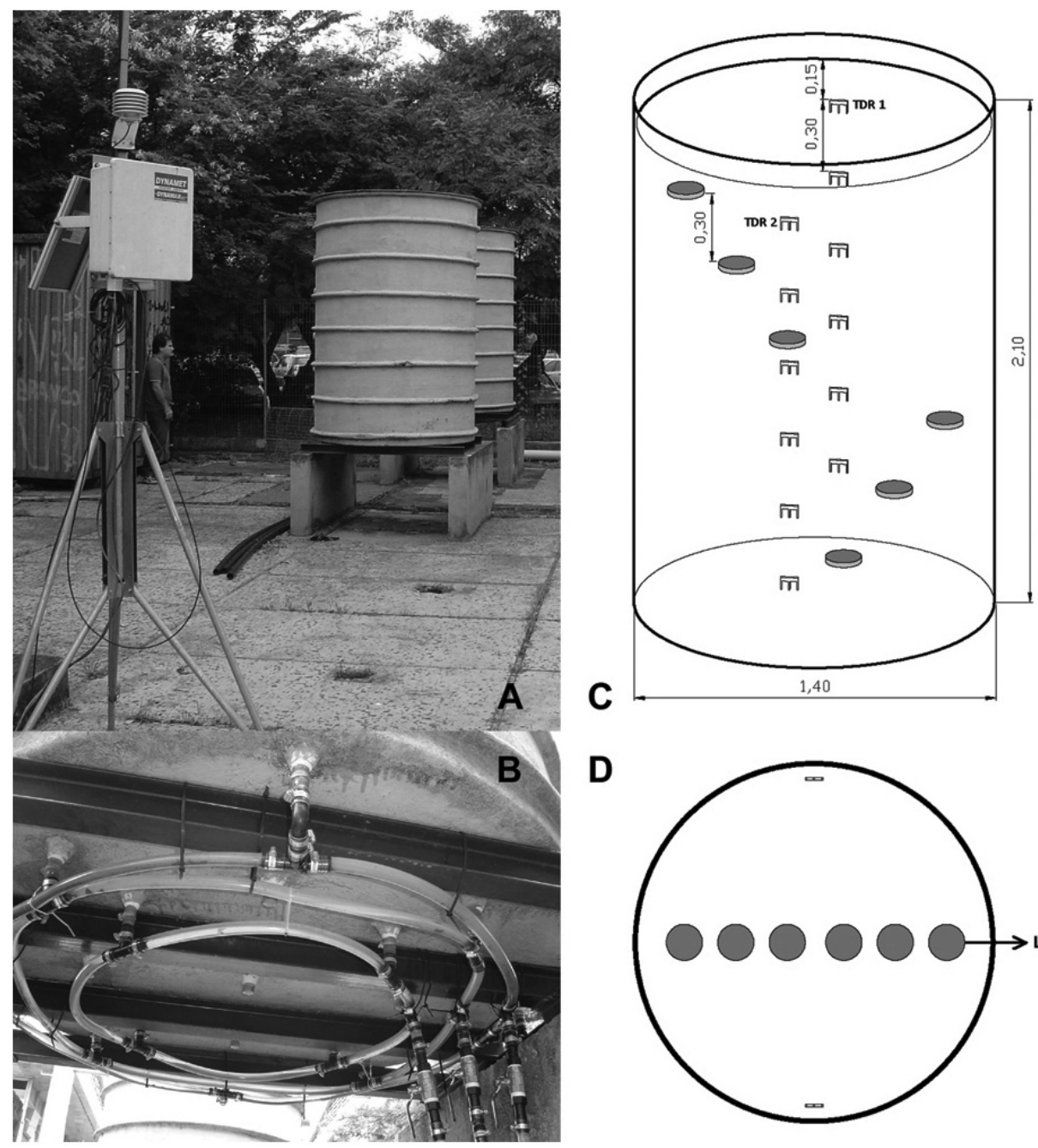

D

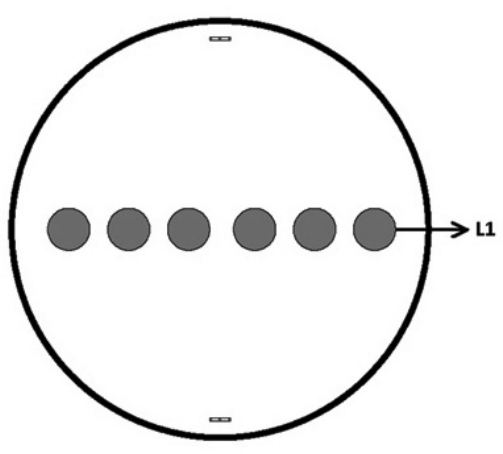

Figura 1 - Características do experimento: A) visão geral do tanque com areia; B) sistema de injeção/drenagem de água pela base; C) representação esquemática da disposição dos TDRs e discos metálicos no tanque com areia; e D) visão da seção horizontal com indicação da linha de aquisição dos perfis de GPR.

Durante o período de aquisição dos dados GPR foram obtidas leituras dos TDR a cada 5 minutos.

A Tabela 1 resume os parâmetros de aquisição empregados no experimento.

\section{Experimento de campo}

As aquisições dos dados foram feitas numa área de escorregamentos no município de Ubatuba, estado de São Paulo. A Figura 2 apresenta a sua localização.

Foram realizados quatro ensaios no decorrer de um ano, em diferentes períodos representativos de regimes pluviométricos distintos (meses de junho, agosto e outubro de 2005 e maio de 2006).

Utilizou-se o sistema RAMAC da Mala Geosciences e antenas biestáticas não blindadas de $200 \mathrm{MHz}$. 0 modo de aquisição em- pregado foi o CMP (Common Mid Point). A Tabela 1 apresenta os parâmetros de aquisição utilizados.

Tabela 1 - Valores de $L$ para os três tipos de diferenças finitas e para diferentes ordens de $d$ e de $p$.

\begin{tabular}{|c|c|c|}
\hline Parâmetro & $\begin{array}{c}\text { Ensaio } \\
\text { laboratório }\end{array}$ & $\begin{array}{c}\text { Ensaio } \\
\text { campo }\end{array}$ \\
\hline frequência central & $1000 \mathrm{MHz}$ & $200 \mathrm{MHz}$ \\
afastamento mínimo & $0,105 \mathrm{~m}$ & $0 \mathrm{~m}$ \\
afastamento máximo & $0,105 \mathrm{~m}$ & $12 \mathrm{~m}$ \\
intervalo espacial de aquisição & $0,02 \mathrm{~m}$ & $0,1 \mathrm{~m}$ \\
janela temporal de aquisição & $40 / 78 \mathrm{~ns}$ & $235 / 421 \mathrm{~ns}$ \\
intervalo de amostragem & $0,077 \mathrm{~ns}$ & $0,46-0,82 \mathrm{~ns}$ \\
empilhamento vertical & $64 / 128$ & 64 \\
\hline
\end{tabular}

Visando avaliar as estimativas feitas com os dados GPR também foram obtidas medidas "pontuais" de teor de umidade 

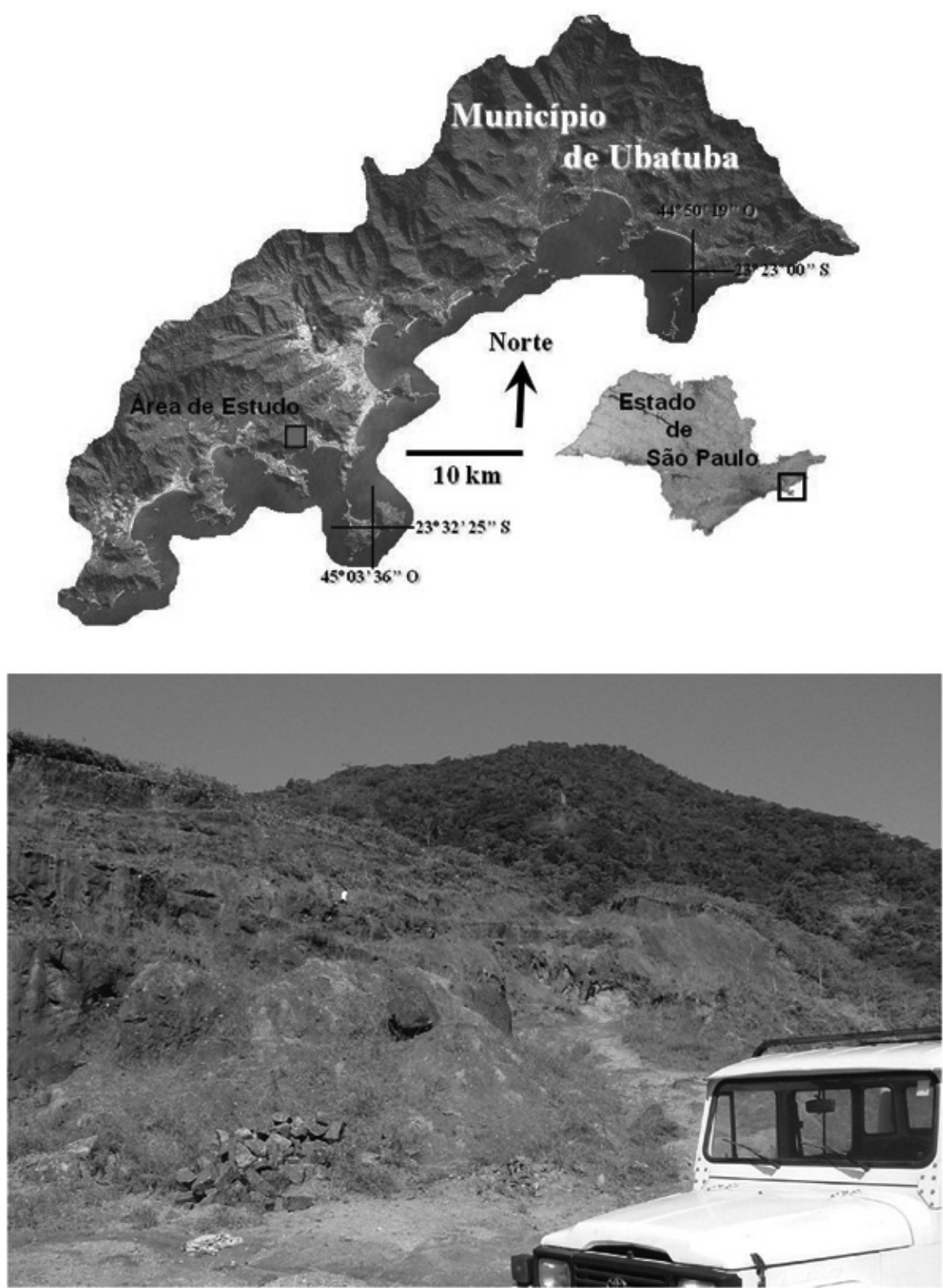

Figura 2 - Localização da área de estudo no município de Ubatuba. Seta indica local dos ensaios (coordenadas UTM N7403242 E488550).

volumétrico a partir de amostras deformadas de solo coletadas com um trado mecânico em seguida à aquisição GPR. Os quatro furos realizados em cada uma das campanhas ficaram próximos entre si, ao final circunscritos a uma área circular com raio aproximado de $0,50 \mathrm{~m}$. Em cada ensaio o furo de trado sempre esteve situado próximo ( $\approx 0,10 \mathrm{~m})$ à posição central do arranjo CMP, sendo que as amostras foram coletadas a cada $0,25 \mathrm{~m}$, até a profundidade máxima de 3,0 m.
As amostras foram hermeticamente embaladas, assim que coletadas. No laboratório foram pesadas e em seguida secadas em uma estufa a $105^{\circ} \mathrm{C}$. Já secas foram novamente pesadas e assim obteve-se a massa de água pela diferença das pesagens. 0 teor de umidade gravimétrico $(w)$ foi obtido pela razão entre a massa de água e a massa da amostra seca. Os teores de umidade volumétricos $(\theta)$ foram obtidos com a Equação (1) onde $\rho_{d}$ representa a massa específica aparente seca. Os valores de 
$\rho_{d}$ foram obtidos por Mendes (2008) de amostras indeformadas.

$$
\theta=w \cdot \rho_{c}
$$

\section{Análise dos dados}

Este trabalho focaliza somente 0 evento relacionado à propagação direta da onda entre as antenas transmissora e receptora. Diferentes estudos já mostraram o potencial do uso da análise da onda direta na avaliação do teor de umidade de meios naturais superficiais (Lesmes et al., 1999; Huisman et al., 2001).

Parte da energia eletromagnética emitida pela antena transmissora transita ao longo da interface ar-solo (onda direta no solo - ground wave). 0 teor de umidade volumétrico calculado através do evento da onda direta traduz a propriedade do solo até uma profundidade específica $\left(d_{i}\right)$, chamada de profundidade de influência. Há discussões sobre a real profundidade de influência na propagação da onda direta (Galagedara et al., 2005). Neste trabalho empregamos a proposta de Van Overmeeren et al. (1997) dada por:

$$
d_{i}=\frac{1}{2} \sqrt{\frac{v s}{f}}
$$

em que $v$ é a velocidade de propagação da onda direta, $s 0$ afastamento entre antenas e $f$ a frequência central da antena empregada.

Os dados GPR foram tratados usando a análise do traço complexo (Taner et al., 1979), especificamente 0 atributo amplitude instantânea (também chamada de envelope do traço). A correlação entre os parâmetros instantâneos, como amplitude, fase e frequência, e a geometria e propriedades físicas do meio é empregada na sísmica do petróleo (Chopra \& Marfurt, 2007).

0 traço GPR registrado num levantamento, $g(t)$, representa a componente real do traço complexo. Empregando o conceito de traço GPR complexo (ou sinal analítico), pode-se obter a parte imaginária, $h(t)$, aplicando-se a transformada de Hilbert ao registro adquirido. Assim o traço complexo GPR, $C(t)$, é dado por:

$$
C(t)=g(t)+i h(t)
$$

Obtido o traço GPR complexo podemos calcular seus atributos como a amplitude instantânea (envelope do traço), fase instantânea e frequência instantânea.

Obtemos a amplitude instantânea da relação:

$$
A(t)=\sqrt{|\operatorname{Re} C(t)|^{2}+|\operatorname{Im} C(t)|^{2}}
$$

A amplitude instantânea assim obtida é independente da fase e se relaciona com a energia registrada ao longo do tempo.
Neste trabalho foram calculados os valores das amplitudes instantâneas ao longo de uma janela temporal restrita ao registro da onda direta, e obtido o valor máximo nesse intervalo.

No caso do ensaio de laboratório adotou-se uma janela constante, de 0 a $10 \mathrm{~ns}$, uma vez que os perfis foram realizados com afastamento fixo das antenas (Tab. 1).

Já para 0 ensaio de campo, que empregou 0 arranjo CMP, fez-se a análise dos traços adquiridos com afastamento de $2 \mathrm{~m}$ e janela temporal de $30 \mathrm{~ns}$ restrita ao registro da onda direta (ground wave). A escolha do afastamento de $2 \mathrm{~m}$ esteve relacionada à busca de uma janela temporal livre das interferências da onda aérea e das eventuais ondas refletidas (Fig. 3).

Para a obtenção da máxima amplitude instantânea dos radargramas dos ensaios de laboratório e de campo empregou-se 0 aplicativo Seismic Unix (Cohen \& Stockwell, 2010).

\section{DESCRIÇÃO DA ÁREA DO ENSAIO DE CAMPO}

A área de estudo está localizada no topo de uma encosta parcialmente degradada por mineração de saibro, nas extremidades de uma serra alongada onde predominam declividades altas (>20\%). Geologicamente esta área é caracterizada pela ocorrência de granulitos parcialmente migmatizados (Mendes, 2008).

As atividades de extração e a posterior erosão geraram um talude vertical com altura média de 10 metros, cujo perfil de alteração pode ser dividido, basicamente, em dois horizontes de solo residual. 0 primeiro (horizonte I-A) possui espessura de aproximadamente 1 metro a partir do topo da encosta natural (onde foram realizados os ensaios), sendo constituído por solo de textura argilo-arenosa de coloração amarela e presença de material orgânico e raízes. 0 segundo (horizonte I-C) possui espessura de 7,0-8,0 metros, sendo formado por saprolito (solo de textura areno-argilo-siltosa e areno-siltosa de coloração variegada, com presença de estrutura reliquiar e apreciável variação de textura, tanto vertical quanto lateralmente).

De modo geral, os horizontes mais superficiais tendem a apresentar maior percentual de argila, enquanto o horizonte I-C, correspondente ao saprolito, tende a apresentar menor porção de argila e maiores porções de areia e silte.

\section{RESULTADOS}

\section{Experimento de laboratório}

Os valores dos máximos das amplitudes instantâneas do dado GPR foram comparados aos dos teores de umidade volumétricos (médias aritméticas) obtidos dos TDRs mais superficiais (TDR1 


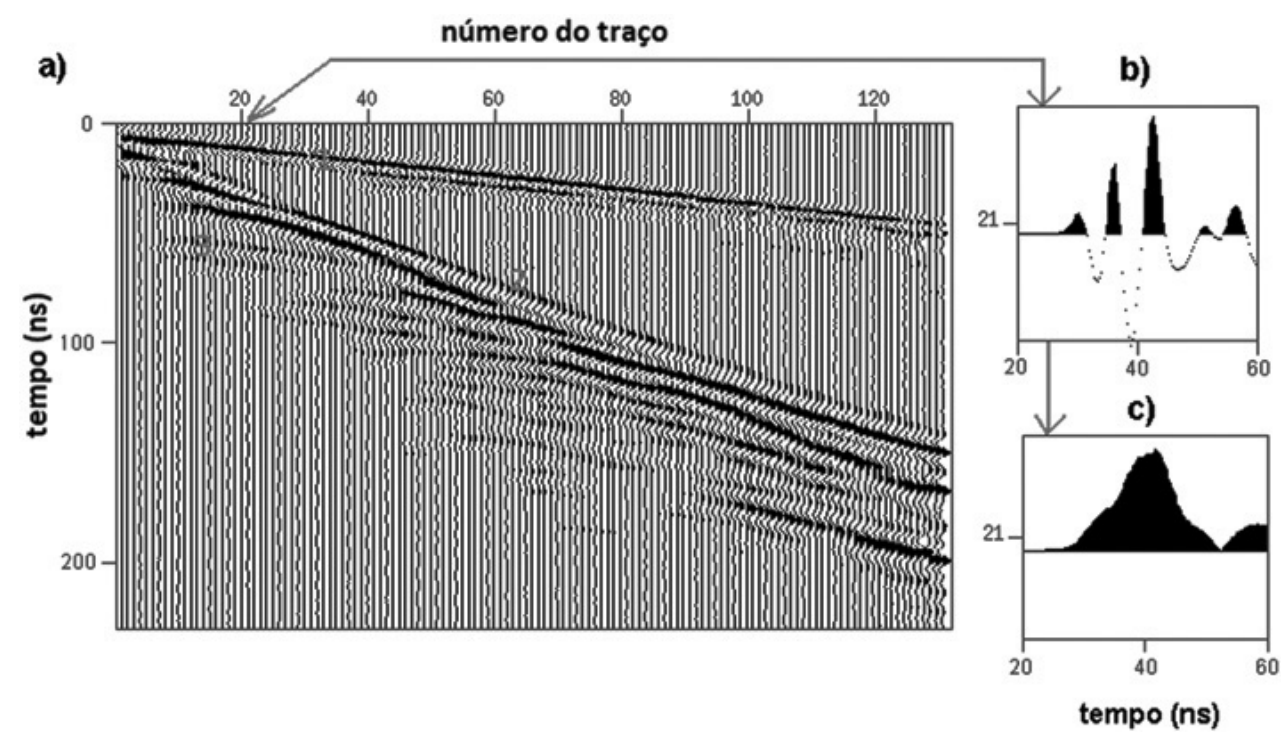

Figura 3 - Esquema ilustrativo dos procedimentos adotados em relação aos radargramas de campo. a) registro CMP completo ( 1 - onda direta; 2 - onda aérea; 3 - onda refletida); b) traço escolhido para análise - afastamento $2 \mathrm{~m}$ e janela temporal envolvendo o registro da onda direta no solo; c) amplitude instantânea da janela escolhida.

e TDR2), posicionados a $0,15 \mathrm{~m}$ de profundidade. A profundidade de influência na propagação da onda direta, neste caso, foi estimada em 0,06 m a partir da Equação (2). Para a obtenção dos valores dos máximos das amplitudes foram empregados os programas suattributes e supickamp do aplicativo Seismic Unix, que realizam a análise dos atributos amplitude, fase ou frequência do traço complexo e a leitura do valor máximo dentro de uma janela específica.

A Figura 4 apresenta os radargramas concatenados que foram adquiridos durante os períodos de enchimento e drenagem (há um hiato de registro durante o período em que o tanque permaneceu saturado), obtidos com a antena fixa e com registros a cada 2 segundos (enchimento) e 1 segundo (drenagem). Pode-se observar a variação do nível de saturação durante as duas operações pela imagem da reflexão nesta interface, sendo que na drenagem a maior atenuação do sinal (devido à água remanescente nos poros do sedimento) torna essa reflexão menos visível. A Figura 5 apresenta os radargramas obtidos ao longo da linha L1 (Fig. 1) no modo afastamento-constante antes do enchimento (Fig. 5A), após a completa saturação (Fig. 5B) e logo após a drenagem (Fig. 5C). As hipérboles de difração que podem ser observadas se relacionam à presença de discos metálicos que foram colocados em posições específicas visando outros experimentos com reflexão de onda (Barbosa et al., 2010).

0 gráfico da Figura $6 \mathrm{~A}$ apresenta os valores dos teores de umidade volumétricos obtidos com os TDRs ao longo do experimento.

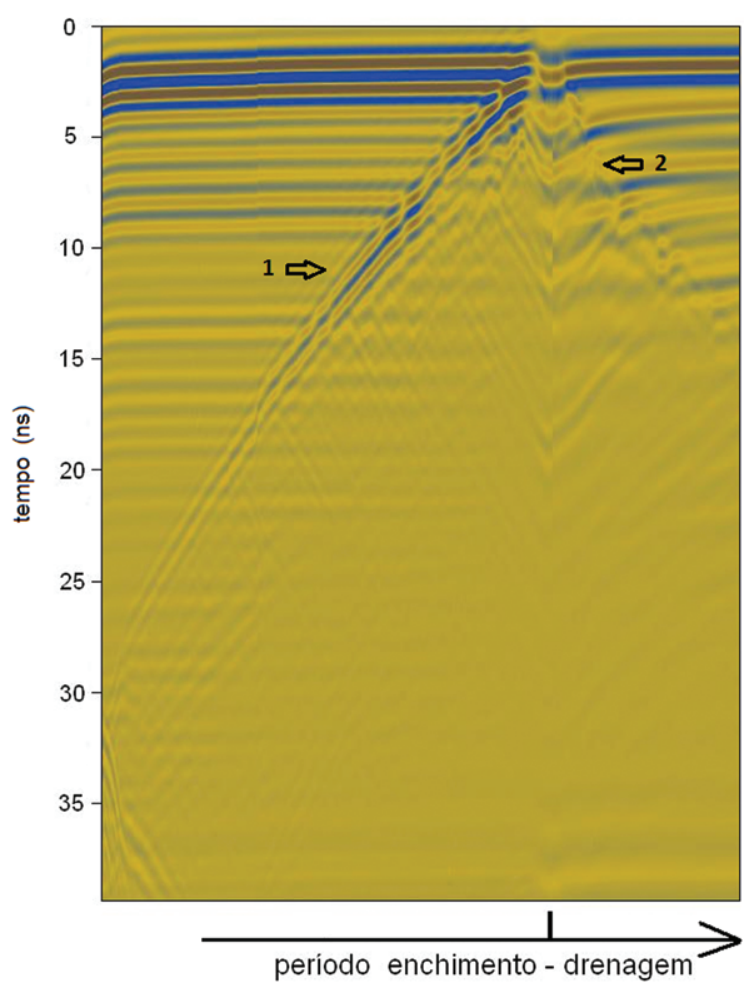

Figura 4 - Radargramas concatenados dos períodos de enchimento e drenagem. Podem ser observadas as reflexões relativas à interface sedimento não saturado-saturado durante 0 enchimento (1) e a drenagem (2).

Durante o período de aquisição dos dados GPR foram obtidas leituras dos TDR a cada 5 minutos. A Figura $6 \mathrm{~A}$ apresenta os dados das leituras dos TDR 1 e 2 , implantados em posições diametralmente opostas e a $0,15 \mathrm{~m}$ de profundidade. Inicialmente 

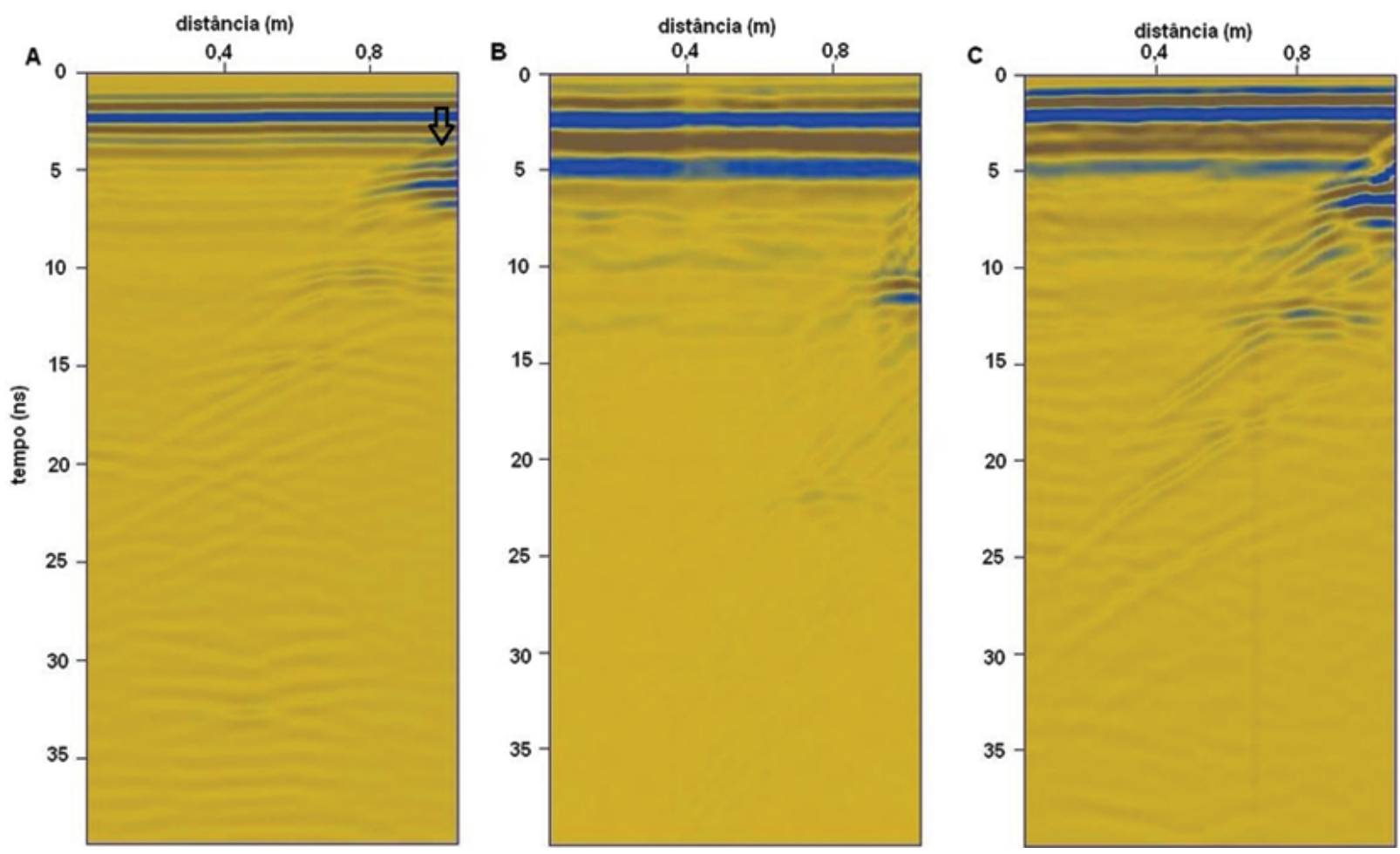

Figura 5 - Seções de GPR obtidas ao longo da linha L1 antes do enchimento (A), durante o período de saturação (B), e após a drenagem (C). A seta indica uma das hipérboles de difração gerada pela presença do disco metálico.

os sedimentos apresentavam teores de umidade volumétrica da ordem de 5\%. Após o enchimento atingiu-se valores próximos a $48 \%$ e durante a drenagem houve um decaimento gradual até valores próximos a $16 \%$.

A Figura $6 \mathrm{~B}$ apresenta os valores dos máximos da amplitude instantânea para os registros contínuos no tempo apresentados na Figura 4 (durante 0 enchimento e a drenagem do tanque) e a média aritmética (triângulo vermelho) das amplitudes obtidas no ensaio ao longo da linha L1 na condição de saturação total do sedimento. Pode-se observar que a resposta em amplitude do sinal GPR sugere uma correlação com os teores de umidade obtidos com os TDRs. Quanto maior o teor de umidade, menor a amplitude do sinal. A mesma resposta foi observada nos dados adquiridos ao longo da linha L1 (Fig. 7). As perturbações de amplitude observadas no início e final da saturação máxima (Fig. 6B) devem estar associadas às mudanças significativas na relação de volume dos fluidos dos poros (ar-água) próximo à superfície, que ocorrem quando da chegada da frente de saturação e no início do seu rebaixamento, alterando momentaneamente os contrastes dielétricos e as condições de acoplamento solo-antena.

A correlação entre os dados GPR e TDR foram limitadas ao período de drenagem do tanque, especificamente no intervalo de 210 a 235 minutos, quando ocorre um decaimento acentuado do teor de umidade. Durante esse período a antena se manteve fixa.

Os valores das amplitudes instantâneas apresentados na Figura 8 representam as médias dos dados obtidos durante dois minutos de aquisição, ou seja, um minuto antes e um após o momento de obtenção da leitura TDR. Os valores dos teores de umidades, por sua vez, representam a média aritmética das leituras obtidas nos TDRs 1 e 2 (Fig. 1).

A Figura 8 mostra que há boa correlação entre os dados, com coeficiente de determinação $\left(R^{2}\right)$ de 0,98 .

\section{Experimento de campo}

Os valores dos máximos das amplitudes instantâneas do dado GPR, obtidos dentro da janela temporal de registro da onda direta, foram comparados aos dos teores de umidade volumétricos obtidos das amostras de trado. A profundidade de influência na propagação da onda direta, no ensaio de campo, foi estimada em 0,6 m a partir da Equação (2). Os valores dos teores de umidade das amostras de campo usados na correlação representam as médias quadráticas (RMS) dos valores obtidos para as amostras coletadas nas profundidades de 0,25 m, 0,50 m e 0,75 m. 
A

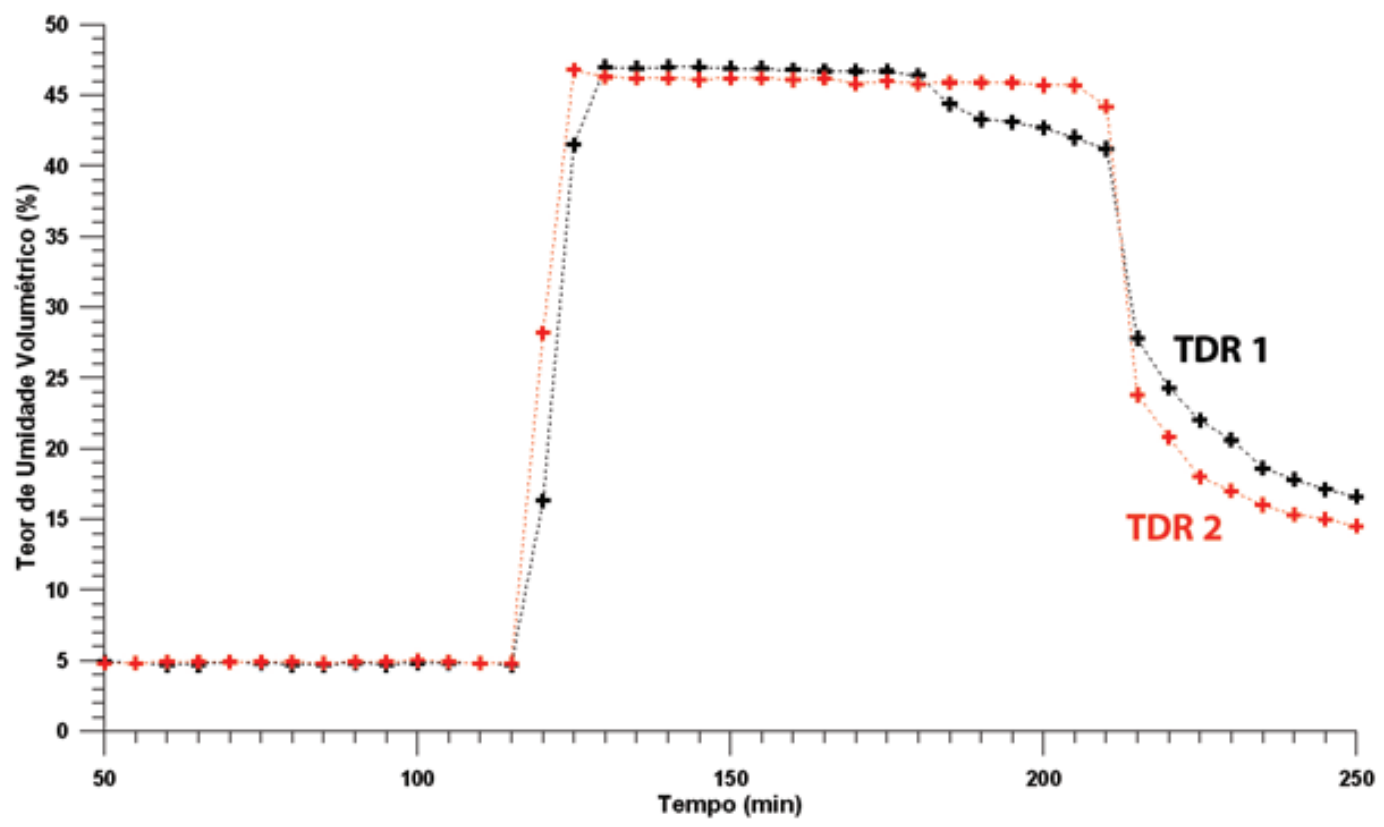

B

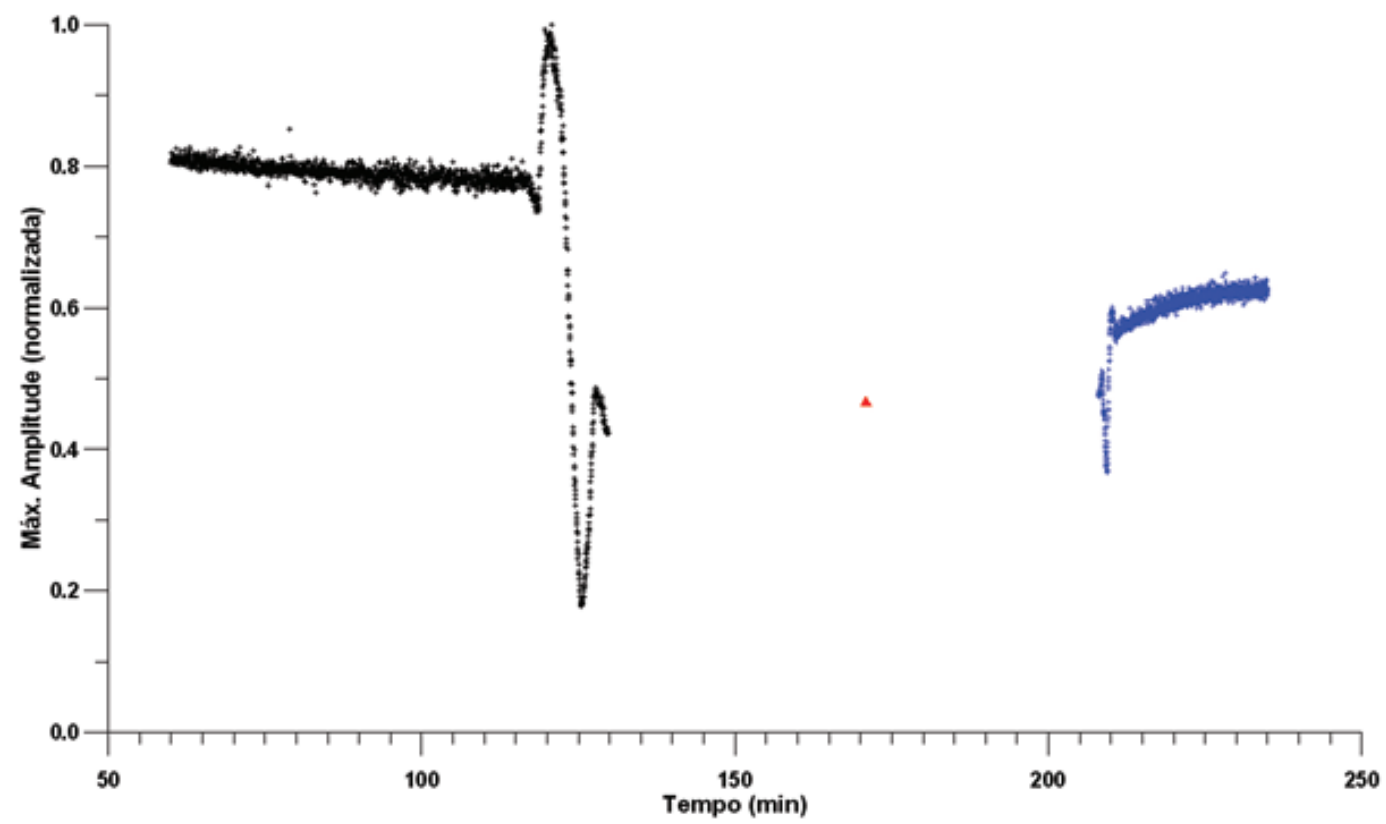

Figura 6 - A) Leituras dos TDRs 1 e 2 (na profundidade de 0,15 m) obtidas durante 0 ensaio GPR (enchimento-drenagem); B) Máximos valores da amplitude instantânea durante 0 enchimento (pontos em preto), na condição de saturação total (triângulo vermelho) e durante a drenagem (pontos em azul). 


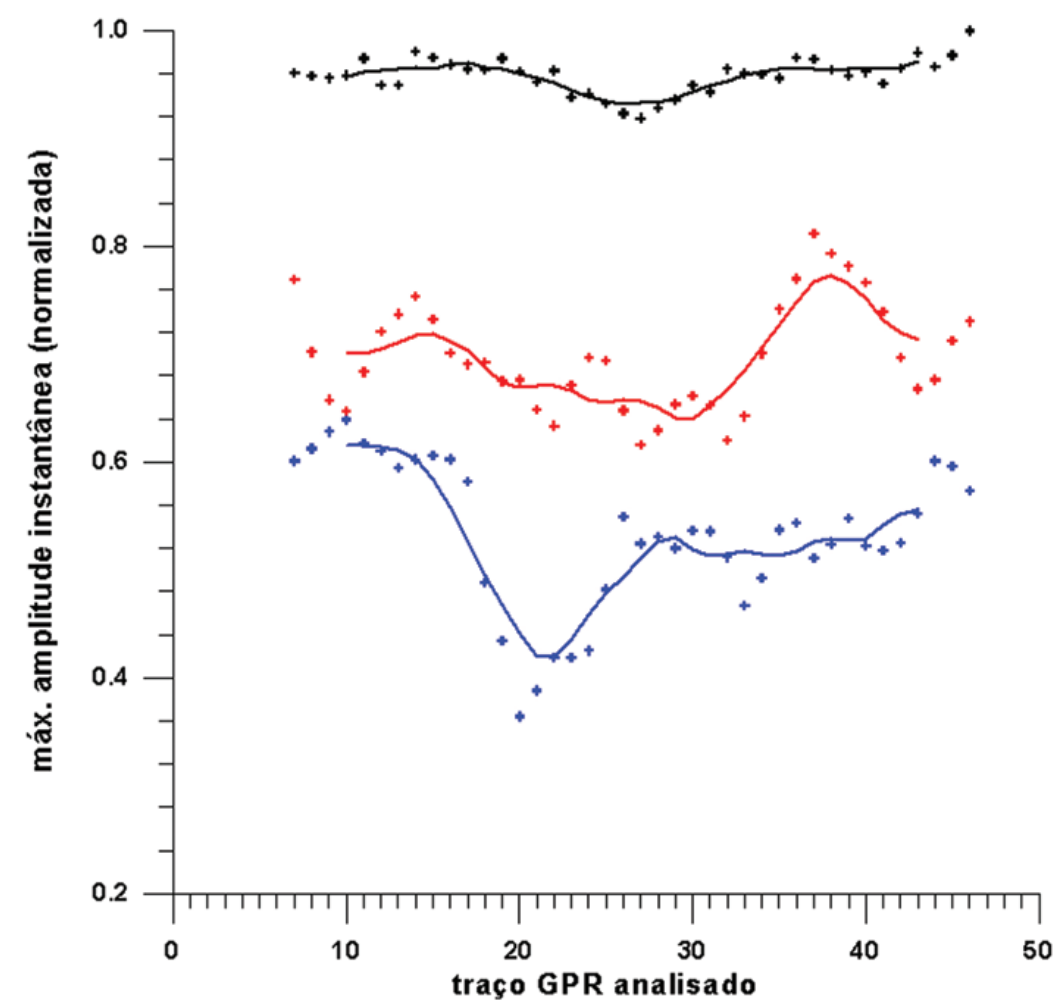

Figura 7 - Valores máximos da amplitude instantânea medidos no perfil L1 antes do enchimento (preto), na condição de máxima saturação (azul) e após a drenagem (vermelho).

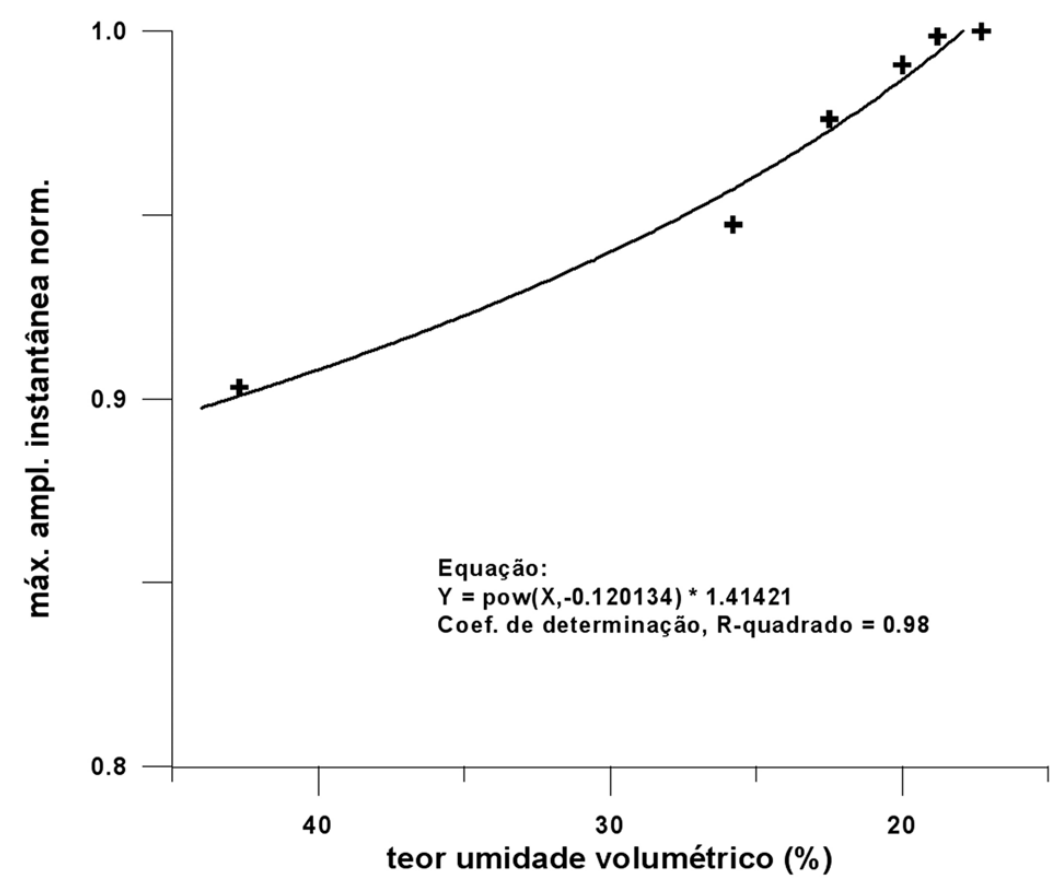

Figura 8 - Correlação entre os dados GPR (amplitude instantânea) e os dados TDR (teor de umidade volumétrico) durante o período de drenagem do tanque. 


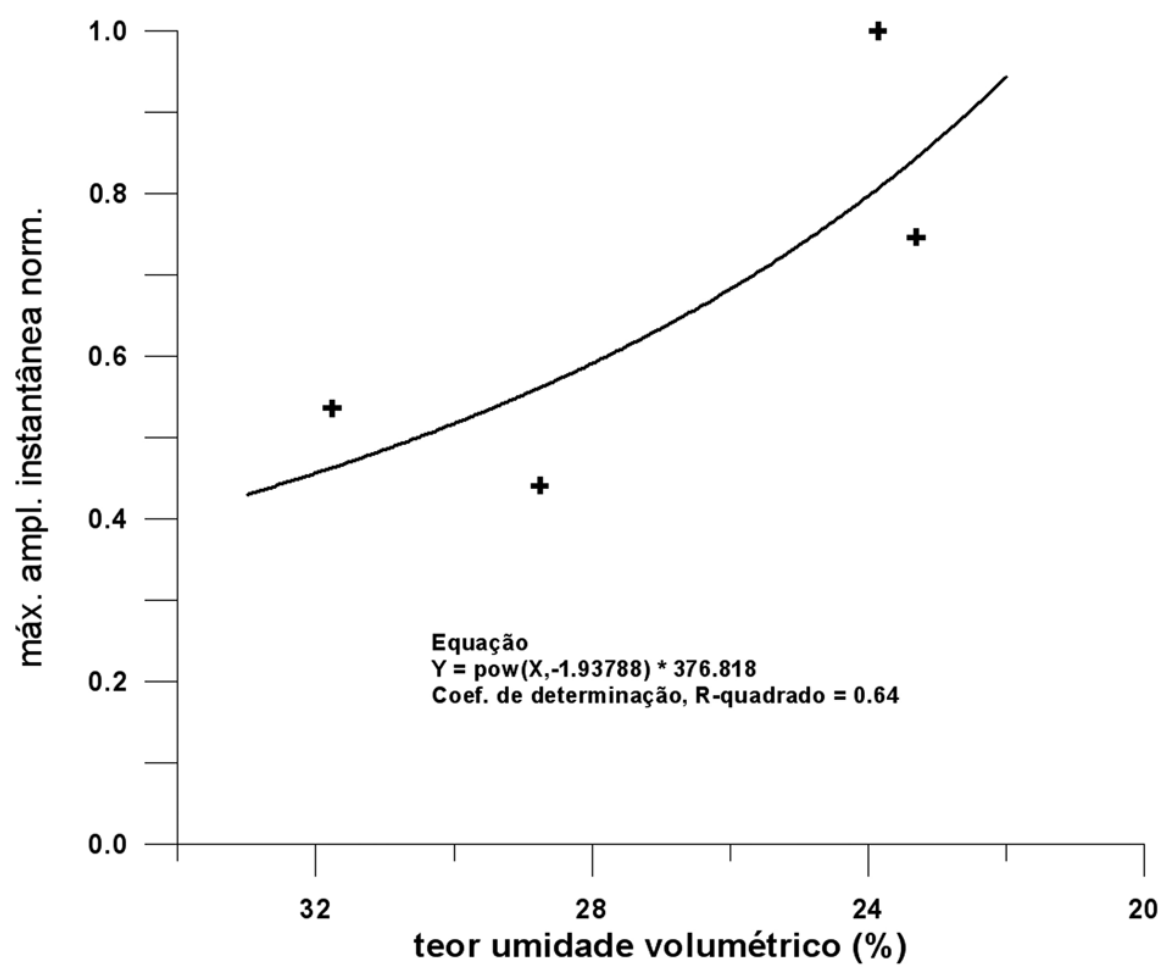

Figura 9 - Correlação entre as amplitudes instantâneas da onda direta registrada em ensaio GPR e as médias RMS das umidades obtidas das amostras coletadas com trado manual (junho, agosto e outubro de 2005 e maio de 2006).

Mesmo tratando-se de dados que foram obtidos em quatro diferentes levantamentos ao longo de um ano, observa-se na Figura 9 que há correspondência entre os valores de amplitude instantânea e os teores de umidade, embora tenha se chegado a um coeficiente de determinação de apenas 0,64. Este valor baixo se deve possivelmente à pequena quantidade de dados utilizados.

\section{CONCLUSÕES E COMENTÁRIOS}

Os experimentos indicaram que há forte correspondência entre os teores de umidade do meio e o parâmetro amplitude instantânea da onda eletromagnética, com potencial, portanto, de emprego deste atributo para avaliações da variação espacial ou temporal do teor de umidade de solos e sedimentos. Para grandes áreas, os dados podem ser rapidamente obtidos usando 0 método GPR com as antenas sendo rebocadas com afastamento constante. 0 afastamento ideal pode ser definido para cada área a ser investigada a partir de um ensaio CMP inicial.

Em condições controladas de laboratório, a correlação apresentou um coeficiente de determinação de 0,98, mas no experimento de campo esse índice foi de 0,64 devido ao pequeno número de dados empregados na correlação, como também às variações nas condições de acoplamento das antenas e às variações laterais do solo verificadas a cada experimento (estas menos importantes uma vez que se procurou o menor deslocamento possível entre cada furo de trado realizado).

As correlações, portanto, devem ser estabelecidas com número estatisticamente significativo de dados.

Terrenos com pronunciadas variações laterais de materiais e porosidade, assim como superfícies muito irregulares, que ocasionem variações no acoplamento das antenas, devem trazer maior imprecisão na avaliação qualitativa das variações dos teores de umidade.

Restringindo-se a investigação por áreas onde não haja, até a profundidade investigada, mudanças significativas dos materiais geológicos supõe-se que a resposta do atributo amplitude instantânea está relacionada preferencialmente ao conteúdo e à salinidade dos fluidos do meio. Deve-se considerar, portanto, 0 caráter sítio-dependente dessas correlações.

0 emprego de diferentes frequências de antenas GPR implica em variações no volume investigado, permitindo assim um zoneamento dos teores de umidade por profundidades.

A metodologia proposta é fácil de ser implementada através de um fluxograma de processamento de rápida execução, e pode ser empregada de maneira complementar à que se utiliza do atributo velocidade. 


\section{AGRADECIMENTOS}

Os autores agradecem à FAPESP (auxílio pesquisa 05/55736-5), ao IAG-USP e à EP-USP pelos apoios recebidos, e aos revisores pelos importantes comentários e observações.

\section{REFERÊNCIAS}

BARBOSA EEM, PRADO RL, MENDES RM \& MARINHO FAM. 2010. Estimativas do teor de umidade empregando 0 método GPR: uma avaliação comparativa em experimentos de laboratório e campo. Revista Brasileira de Geofísica, 28(4): 691-701.

CHARLTON M. 2000. Small scale soil-moisture variability estimated using ground penetrating radar. Extended abstract. In: Proceedings of the Eighth International Conference on Ground Penetrating Radar, Gold Coast, Australia, 23-26 May. CD-ROM.

CHOPRA S \& MARFURT KJ. 2007. Seismic attributes for prospect identification and reservoir characterization. Geophysical Developments (11). Society of Exploration Geophysicists. 464 p.

COHEN JK \& STOCKWELL JJW. 2010. CWP/SU: Seismic Unix Release 41: a free package for seismic research and processing. Center for Wave Phenomena, Colorado School of Mines. $135 \mathrm{p}$.

CORBEANU RM, McMECHAN GA, SZERBIAK RB \& SOEGAARD K. 2002. Prediction of 3-D fluid permeability and mudstone distributions from ground-penetrating radar (GPR) attributes: Example from the Cretaceous Ferron Sandstone Member, east-central Utah. Geophysics, 67: 1495-1504.

GALAGEDARA LW, REDMAN JD, PARKIN GW, ANNAN AP \& ENDRES AL. 2005. Numerical modeling of GPR to determine the direct ground wave sampling depth. Vadose Zone Journal, 4: 1096-1106.

GROTE K, HUBBARD S \& RUBIN Y. 2003. Field-scale estimation of volumetric water content using GPR ground wave techniques. Water Resources Research, 39(11): 1321, doi: 10.1029/2003WR002045.

HUISMAN JA, SPERL C, BOUTEN W \& VERSTRATEN JM. 2001. Soil water content measurements at different scales: accuracy of time domain reflectometry and ground-penetrating radar. Journal of Hydrology, 245: $48-58$.

HUISMAN JA, HUBBARD SS, REDMAN JD \& ANNAN AP. 2003. Measuring soil water content with ground penetrating radar: A review. Vadose Zone Journal, 2: 476-491.

KIM J, JEONG S, PARK S \& SHARMA J. 2004. Influence of rainfallinduced wetting on the stability of slopes in weathered soils. Engineering Geology, 75: 251-262.

LESMES D, HERBSTZUBER RJ \& WERTZ D. 1999. Terrain permittivity mapping: GPR measurements of near-surface soil moisture. In: Symp. Appl. Geophys. Eng. Environ. Prob., Proceedings, EEGS, p. 575-582.

MENDES RM. 2008. Estudo das propriedades geotécnicas de solos residuais não saturados de Ubatuba (SP). Tese (Doutorado em Engenharia), Escola Politécnica, Universidade de São Paulo, São Paulo, 236 p.

PETTINELLI E, VANNARONI G, DI PASQUO B, MATTEI E, DI MATTEO A, DE SANTIS A \& ANNAN PA. 2007. Correlation between near-surface electromagnetic soil parameters and early-time GPR signals: An experimental study. Geophysics, 72(2): 25-28.

SCHMALZ B, LENNARTZ B \& WACHSMUTH D. 2002. Analyses of soil water content variations and GPR attribute distributions. Journal of Hydrology, 267: 217-226.

TANER MT, KOCHELER F \& SHERIFF RE. 1979. Complex seismic trace analysis. Geophysics, 44(6): 1041-1063.

TOPP GC, DAVIS JL \& ANNAN AP. 1980. Electromagnetic determination of soil water content: Measurements in coaxial transmission lines. Water Resources Research, 16: 574-582.

VAN OVERMEEREN RA, SARIOWAN SV \& GEHRELS JC. 1997. Ground penetrating radar for determining volumetric soil water content; results of comparative measurements at two test sites. Journal of Hydrology, 197: 316-338.

\section{NOTAS SOBRE OS AUTORES}

Renato Luiz Prado. Bacharel em Geologia (IGc/USP, 1981), mestre em Geofísica (IAG/USP, 1994) e doutor em Geociências e Meio Ambiente (IGCE/UNESP, 2000). Professor do IAG, Universidade de São Paulo.

Emílio Eduardo Moreira Barbosa. Bacharel em Geofísica (IAG/USP, 2009). Mestre em Geofísica (IAG-USP, 2011).

Rodolfo Moreda Mendes. Engenheiro Civil (FEIS-UNESP - Ilha Solteira, 1997), mestre em Engenharia Urbana (UFSCar, 2001) e doutor em Engenharia Geotécnica (EP/USP, 2008). Pesquisador cientííico do Instituto Geológico do Estado de São Paulo.

Fernando Antônio Medeiros Marinho. Engenheiro Civil (UFPE, 1983), mestre em Mecânica dos Solos (PUC-RJ, 1986), doutor em Geotecnia (University of London, 1994) e pós-doutorado (Arizona State University, 2008). Professor Associado da Escola Politécnica da USP.

Orlando Martini de Oliveira. Engenheiro Civil (UFPE, 1995), mestrado em Engenharia Civil (UFPE, 1999), doutorado e pós-doutorado (USP, 2004 e 2006). Professor Adjunto da Universidade Federal de Santa Catarina, desde 2006 